\title{
Détermination des paramètres optimaux d'exposition aux rayons $X$ en microdensitométrie du bois
}

\author{
P. DUPONT, J. FAGOT, M. LEUSCH, J.-M. GODEFROID, \\ A. CULOT, L. FRAIPONT \\ Chaire de Technologie forestière \\ Faculté des Sciences Agronomiques de l'Etat, 5800 Gernbloux, Belgique
}

\author{
Résumé
}

Cette étude définit, puis formalise la notion d'exposition optimale compte tenu des contraintes techniques rencontrées dans l'analyse microdensitométrique d'échantillons de bois. De plus, elle montre, par un exemple, qu'il est facile, empiriquement, de rechercher cet optimum, dans chaque cas particulier, en jouant sut la tension aux bornes du tube, le temps d'exposition et (ou) le courant de chauffage de la cathode.

Mots clés: Bois, conditions d'exposition, densité, rayons $X$, microdensitométrie.

\section{Introduction}

Les paramètres d'exposition aux rayons $\mathrm{X}$ des échantillons de bois soumis à l'analyse microdensitométrique (PoLgE, 1966) varient de manière importante d'un laboratoire à l'autre (EVERTSEN, 1982).

Ces différences sont dues principalement au type de film, à la source de radiation, à la distance source-film et à l'épaisseur des échantillons. Nous voulons présenter ici, non seulement les conditions et le matériel d'exposition que nous utilisons au laboratoire de microdensitométrie de Gembloux, mais surtout la méthode qui nous a conduits au choix des paramètres (tension, intensité et temps).

\section{Type de film}

Selon l'enquête réalisée par EverTsen (op. cit.), les films utilisés dans les différents laboratoires se distinguent par leur rapidité et par leur structure (monoface ou double face). Cependant, il s'agit toujours de films de marque Kodak.

Dans notre laboratoire, nous utilisons des films Structurix D4 de la marque AgfaGevaert. Ceux-ci, de format $18 \times 24 \mathrm{~cm}$, possèdent un grain très fin ; leur effet contrastant est élevé et leur rapidité moyenne. 


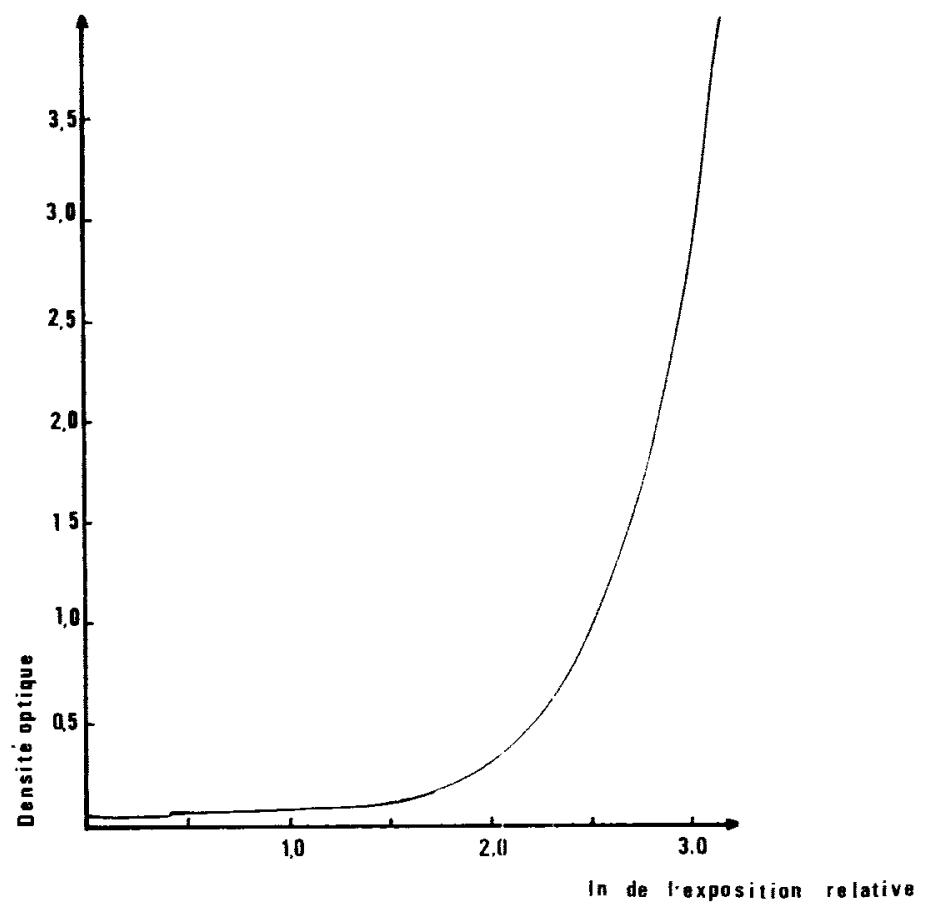

Fig. I

Courbe sensitométrique du film Structurix D4 exposé aux rayons $X$ (AgFa-GeVAERT, 1969).

Sensitometric curve of the radiofilm Structurix D4 exposed to X-rays (AGFA-GEVAERT, 1969).

Nous présentons à la figure 1 la courbe sensitométrique de ce film telle qu'elle est fournie par le fabricant (AgFa-GEvaERT, 1969). D'après cette courbe, on peut constater que la relation entre l'exposition (I) et la densité optique (D) est, pour D compris entre 0,5 et 2,5 en tout cas, exactement du type :

$$
\mathrm{D}=\mathrm{ae}^{\mathrm{bin} !}
$$

ou

$$
\ln \mathrm{D}=\ln \mathrm{a}+\mathrm{b} . \ln \mathrm{I}
$$

Ce phénomène semble général pour les émulsions radiographiques exposées aux rayons $\mathrm{X}$ (Polge, 1966).

\section{Source de radiation}

Comme les autres laboratoires, nous utilisons des rayons $\mathrm{X}$ à grande longueur d'onde émis, dans notre laboratoire à Gembloux, par un tube radiogène à fenêtre de béryllium (de $1,5 \mathrm{~mm}$ d'épaisseur monté dans une gaine hermétique isolée à l'huile (Machlett OEG 60). Le refroidissement de l'anode se fait par circulation d'eau. La 
tension aux bornes du tube et le courant cathodique ont respectivement pour maximum $50 \mathrm{kV}$ et $40 \mathrm{~mA}$ pour un foyer de $6 \times 6 \mathrm{~mm}$.

\section{Distance de radiation}

Nous avons choisi pour des raisons pratiques une distance source-film de $2,1 \mathrm{~m}$ (DUPONT, 1978). Il semble que cette distance soit aussi celle adoptée par les autres laboratoires : 2 à $2,5 \mathrm{~m}$ selon Evertsen (op. cit.).

\section{Paramètres d'exposition}

Nous avons rappelé dans l'introduction les principales sources de variation pouvant expliquer les valeurs très différentes choisies par les laboratoires pour la tension aux bornes du tube $(10$ à $35 \mathrm{kV})$, le courant de chauffage de la cathode (5 à $21 \mathrm{~mA}$ ) et le temps d'exposition ( 1 minute à plusieurs heures) (Evertsen, op. cit. ; Polge, op. cit.). En radiographie le temps et l'intensité jouent un rôle approximativement équivalent. Une fois fixé le type de matériel employé, la détermination des paramètres d'exposition ne peut être faite que de manière empirique.

L'objectif est d'obtenir un contraste et un noircissement tels que la gamme de mesure de microdensitomètre couvre la gamme des densités optiques correspondant à la gamme de variation de masse volumique du bois, et ce, pour chaque épaisseur d'échantillons.

Les paramètres d'exposition sont modifiés pour atteindre cet objectif : une augmentation du temps d'exposition augmente le contraste, une diminution de la tension permet de ramener la densité optique maximale à un niveau acceptable. Un autre choix aurait pu être proposé, pour arriver au même résultat, à savoir : une diminution importante de la tension (pour enrichir le spectre en grandes longueurs d'ondes) jointe à une augmentation du temps d'exposition.

\subsection{Recherche du contraste optimum}

Augmenter le contraste, c'est traduire une même différence de masse volumique par un plus grand écart de densité optique de manière à augmenter la précision des mesures.

L'optimum ne correspond cependant pas à un contraste trop élevé. En effet, pour un réglage donné, le microdensitomètre, équipé du coin optique linéaire ${ }^{(*)}$ dont nous disposons, peut enregistrer simultanément des densités optiques distantes de 2 unités au maximum.

$\left(^{*}\right)$ Il faut savoir que du fait des lois de noircissement des émulsions radiographiques, les déplacements des coins linéares (i.c. dont les densités optiques varient linéairement d'une extrémité à lautre) ne sont pas reliés linćairement aux variations de la densité du bois. 


\subsection{Gamme de mesure du microdensitomètre}

Notre microdensitomètre fournit une réponse correcte jusqu'à des densités optiques de 3. Certains peuvent aller jusqu'à 6 .

Cependant, pour que les radiographies restent aisément observables à l'œil, cette possibilité ne nous semble pas intéressante et il nous paraît préférable de fixer la densité optique maximale à 2,5 .

La gamme utile de densité optique pour une analyse s'étend donc de 0,5 à 2,5 (voir 5.1.).

\subsection{Gamme de variation de la masse volumique du bois}

De manière à définir les paramètres d'exposition pour un large éventail d'essences, nous avons retenu les valeurs suivantes:

Masse volumique minimale possible : $0,1 \mathrm{~kg} / \mathrm{dm}^{3}$.

Masse volumique maximale possible : $1,3 \mathrm{~kg} / \mathrm{dm}^{3}$.

\subsection{Epaisseur des échantillons}

Le choix de l'épaisseur de l'échantillon est conditionné par ses caractéristiques propres (hétérogénéité, largeurs d'accroissement, et orientation du fil) et par la méthode microdensitométrique (Polge, op. cit).

Selon le cas, les échantillons auront de 1,5 à $20 \mathrm{~mm}$ d'épaisseur. Pour nos besoins, nous avons retenu : 1,$5 ; 3$ et $6 \mathrm{~mm}$.

\subsection{Conclusions}

Des paragraphes précédents, il découle que nous devons chercher expérimentalement pour chacune des épaisseurs choisies, la tension $(\mathrm{kV})$, le courant cathodique $(\mathrm{mA})$ et le temps d'exposition (minute), de manière à ce qu'aux masses volumiques extrêmes $\left(0,1 \mathrm{~kg} / \mathrm{dm}^{3}\right.$ et $\left.1,3 \mathrm{~kg} / \mathrm{dm}^{3}\right)$ correspondent les densités optiques extrêmes $(2,5 \mathrm{D}$ et $0.5 \mathrm{D})$.

Le paragraphe suivant décrit la méthode utilisée pour y parvenir et donne les résultats obtenus pour nos conditions d'exposition.

\section{Expérimentation}

\subsection{Bases theroriques}

On a (Polge, op. cit.), pour un rayonnement $X$ d'intensité incidente $I_{o}$ traversant un matériau dont le coefficient d'absorption massique est $\mu_{m}$, l'épaisseur $x$ et la masse volumique $\mathrm{d}$ :

$$
\mathrm{I}=\mathrm{I}_{0} \mathrm{e}^{-\mu_{\mathrm{m} x \mathrm{~d}}}
$$

où I est l'intensité du rayonnement transmis. 
L'équation (3) peut encore se mettre sous la forme

$$
\ln \mathrm{I}=\ln \mathrm{I}_{\mathrm{o}}-\mu_{\mathrm{m}} \mathrm{Xd}
$$

En remplaçant 1 In I par cette valeur dans l'équation (2) du $\S 2$, on obtient :

$$
\ln \mathrm{D}=\ln \mathrm{a}+\mathrm{b} \ln \mathrm{I}_{\mathrm{o}}-\mathrm{b} \mu_{\mathrm{m}} \mathrm{xd}
$$

ou, en posant :

et

$$
\begin{gathered}
\ln a+b \ln I_{o}=\ln a^{\prime} \\
-b \mu_{m}=b^{\prime} \\
\text { in } D=\ln a^{\prime}+b^{\prime} x d \\
D=a^{\prime} e^{b^{\prime} x d}
\end{gathered}
$$

on a

relation qui lie de manière exponentielle et pour une épaisseur $\mathrm{x}$ constante, la densité optique $\mathrm{D}$ à la masse volumique $\mathrm{d}$ du matériau pour autant que l'on travaille avec l'émulsion décrite au $\S 2$ et dans des densités optiques comprises entre 0,5 et 2,5 (conditions suffisantes).

En exprimant la masse volumique en $\mathrm{kg} / \mathrm{dm}^{3}$, l'équation (6) est celle d'une droite qui doit passer par les points de coordonnés $(0,1 ; \ln 2,5)$ et $(1,3 ; \ln 0,5)$, les abscisses correspondant aux masses volumiques du bois extrêmes et les ordonnées, aux logarithmes des densités optiques extrêmes.

Cette droite a pour coefficient angulaire :

$$
b^{\prime} x=-1,341
$$

et pour ordonnée à l'origine :

$$
\ln \mathrm{a}^{\prime}=1,050
$$

Rechercher les paramètres d'exposition, c'est donc déterminer expérimentalement la combinaison courant cathodique-temps-tension qui fournira ces valeurs de coefficient angulaire et d'ordonnée à l'origine.

Pour augmenter la valeur du coefficient angulaire (le contraste), il faut augmenter le temps d'exposition ou le courant cathodique tout en diminuant la tension aux bornes du tube, pour maintenir le noircissement à un niveau acceptable.

\subsection{Réalisation pratique}

Pour chaque essai d'exposition, la détermination des paramètres $a^{\prime}$ et $b^{\prime}$ peut se faire par la méthode des moindres carrés appliquée à différents couples de points (d, In D) couvrant aussi complètement que possible les gammes de variation fixées précédemment $(\$ 5.2$. et 5.3 .).

En pratique, nous avons fait varier l'épaisseur $x$ plutôt que la masse volumique $d$ en radiographiant un étalon d'acétate de cellulose en escalier. $x$ et $d$ jouent, en effet, un rôle analogue car la relation (6) lie In $\mathrm{D}$ et le «poids superficiel » $\mathrm{dx}$ (Polge, op. cit.).

De plus, le temps jouant le même rôle que l'intensité sur la dose reçue et donc le noircissement, nous avons décidé, pour des raisons d'appareillage, de travailler à intensité constante $(24 \mathrm{~mA})$ et de faire varier seulement le temps d'exposition. 


\subsection{Avantages du modèle théorique du paragraphe 6.1. pour le calcul de la masse volumique}

Alors que nous avons travaillé précédemrnent avec un modèle de transformation linéarisé par l'utilisation d'un coin optique à variation non linéaire, comme beaucoup d'autres laboratoires, nous avons choisi de nous baser sur ce modèle théorique $(\$ 6.1$.) et ce, pour plusieurs raisons :

- Ce modèle est directement basé sur les équations répondant le plus exactement aux phénomènes physiques qui interviennent, à savoir la réaction du film au rayonnement et l'atténuation du rayonnement transmis en fonction du "poids superficiel " et du coefficient d'absorption. Ces lois sont valides pour toute la gamme de densités optiques convenables pour le microdensitomètre. Nous n'avons donc aucun biais des mesures par des lois seulement approximatives, ni aucune perte de précision par une limitation trop importante de la plage de densités optiques à utiliser.

- D'autre part, ce modèle permet l'utilisation du coin optique linéaire, fourni d'origine avec l'appareillage, plus précis et plus stable dans le temps que les coins non linéaires fabriqués par radiographie (voir le point 5.1.).

— Le modèle « linéarisé » utilisé précédemment comportait l'avantage de la simplicité des calculs de transformation. Les moyens de traitement en série de l'information dont nous disposons actuellement rendent cet avantage négligeable.

\subsection{Exemple}

Pour faciliter la compréhension de la méthode utilisée, nous pensons qu'il est utile de présenter ci-dessous un essai complet. Les paramètres d'exposition à tester sont les suivants, pour un échantillon de bois de $3 \mathrm{~mm}$ d'épaisseur, un film Structurix D4 et une distance source-objet de $2,1 \mathrm{~m}$ :

$$
\begin{array}{ll}
\text { temps } & : 25 \mathrm{~min} \\
\text { tension }: & 15 \mathrm{kV} \\
\text { intensité } & : 24 \mathrm{~mA} .
\end{array}
$$

La réalisation du test se fait en radiographiant un étalon d'acétate de cellulose $\left(1,310 \mathrm{~kg} / \mathrm{dm}^{3}\right)$ constitué de 10 marches de 5 feuilles d'acétate (épaisseur d'une feuille : $0,06 \mathrm{~mm})$.

L'équivalence masse volumique-bois de chaque palier est obtenu de la manière suivante :

$$
\mathrm{d}_{\mathrm{h}}=\frac{\mathrm{xa} \mathrm{da}}{\mathrm{x}_{\mathrm{h}}}
$$

$x_{i 1}$ et $d_{i t}$ sont respectivement l'épaisseur et la rnasse volumique de l'acétate,

$x_{b}$ et $d_{b}$, l'épaisseur et la masse volumique du bois.

Notre microdensitomètre étant équipé d'un coin optique linéaire, la densité optique de chaque palier est obtenue par une régression linéaire passant par deux points étalons de densités optiques 1 et 1,5 .

Les résultats sont repris dans le tableau 1. La gamme de densité optique de ce coin (voir \$5.1.) ne nous a pas permis d'analyser 10 paliers simultanément. L'ajustement linéaire entre d et ln $\mathrm{D}$ se calcule donc sur la base des paliers 2 à 10. 


\section{Tableau 1}

Résultats d'analyse densitométrique d'un étalon en acétate de cellulose comprenant dix paliers d'épaisseur.

The densitometric analyse's results of a ten-step cellulose acetate standard.

\begin{tabular}{c|c|c|c|c|c}
\hline \hline Paliers & $\mathrm{x}_{\mathrm{a}}$ & $\mathrm{ps}$ & $\mathrm{d}_{\mathrm{b}}$ & $\mathrm{D}$ & $\ln \mathrm{D}$ \\
\hline 1 & 0,30 & 0,393 & 0,131 & - & - \\
2 & 0,60 & 0,786 & 0,262 & 2,080 & 0,732 \\
3 & 0,90 & 1,179 & 0,393 & 1,716 & 0,540 \\
4 & 1,20 & 1,572 & 0,524 & 1,419 & 0,350 \\
5 & 1,50 & 1,965 & 0,655 & 1,184 & 0,169 \\
6 & 1,80 & 2,358 & 0,786 & 0,990 & $-0,010$ \\
7 & 2,10 & 2,751 & 0,917 & 0,843 & $-0,171$ \\
8 & 2,40 & 3,144 & 1,048 & 0,704 & $-0,351$ \\
9 & 2,70 & 3,537 & 1,179 & 0,606 & $-0,501$ \\
10 & 3,00 & 3,930 & 1,310 & 0,526 & $-0,642$ \\
\hline
\end{tabular}

(1) $x_{i 1}$ : épaisseur d'acétate (densité 1,31 ).

(2) ps: poids superficiel $=x_{a} d_{a l}$ en $\mathrm{kg} / \mathrm{m}^{2}$.

(3) $\mathrm{d}_{\mathrm{b}}$ : masse volumique du bois ( $3 \mathrm{~mm}$ d'ćpaisscur) en $\mathrm{g} / \mathrm{cm}^{3}$.

(4) D : densité optique.

L'équation obtenue par la méthode des moindres carrés est la suivante :

$\ln \mathrm{D}=1,049-1,318 \mathbf{d}_{\mathrm{h}}$

avec un coefficient de détermination $\left(\mathrm{r}^{2}\right)$ de 0,998 . $\S 6.1$.

La figure 2 représente cette droite ainsi que la droite d'exposition idéale définie au

Les deux droites étant presque confondues, nous pouvons accepter les valeurs testées $(24 \mathrm{~mA}, 15 \mathrm{kV}, 25 \mathrm{~min})$ comme paramètres d'exposition. Si cela n'avait pas été le cas ou si on voulait une coüncidence parfaite entre la droite observée et la droite idéale, il faudrait dans ce cas, augmenter le temps d'exposition (pour que le coefficient angulaire passe de $-1,318$ à $-1,341$ ) et diminuer la tension de manière à maintenir l'ordonnée à l'origine à sa valeur.

\section{Résultats}

Cette méthode expérimentale nous a permis de fixer les paramètres d'exposition repris dans le tableau 2 .

Il est à noter que pour des épaisseurs de $1,5 \mathrm{~mm}$, la droite d'exposition obtenue dans ces conditions est moins contrastée que la droite théorique (coefficient angulaire de l'ordre de $-1,15$ au lieu de $-1,34$ ). Nous avons accepté cette différence pour ne pas augmenter trop fortement la durée qui est déjà de plus de 6 heures. 


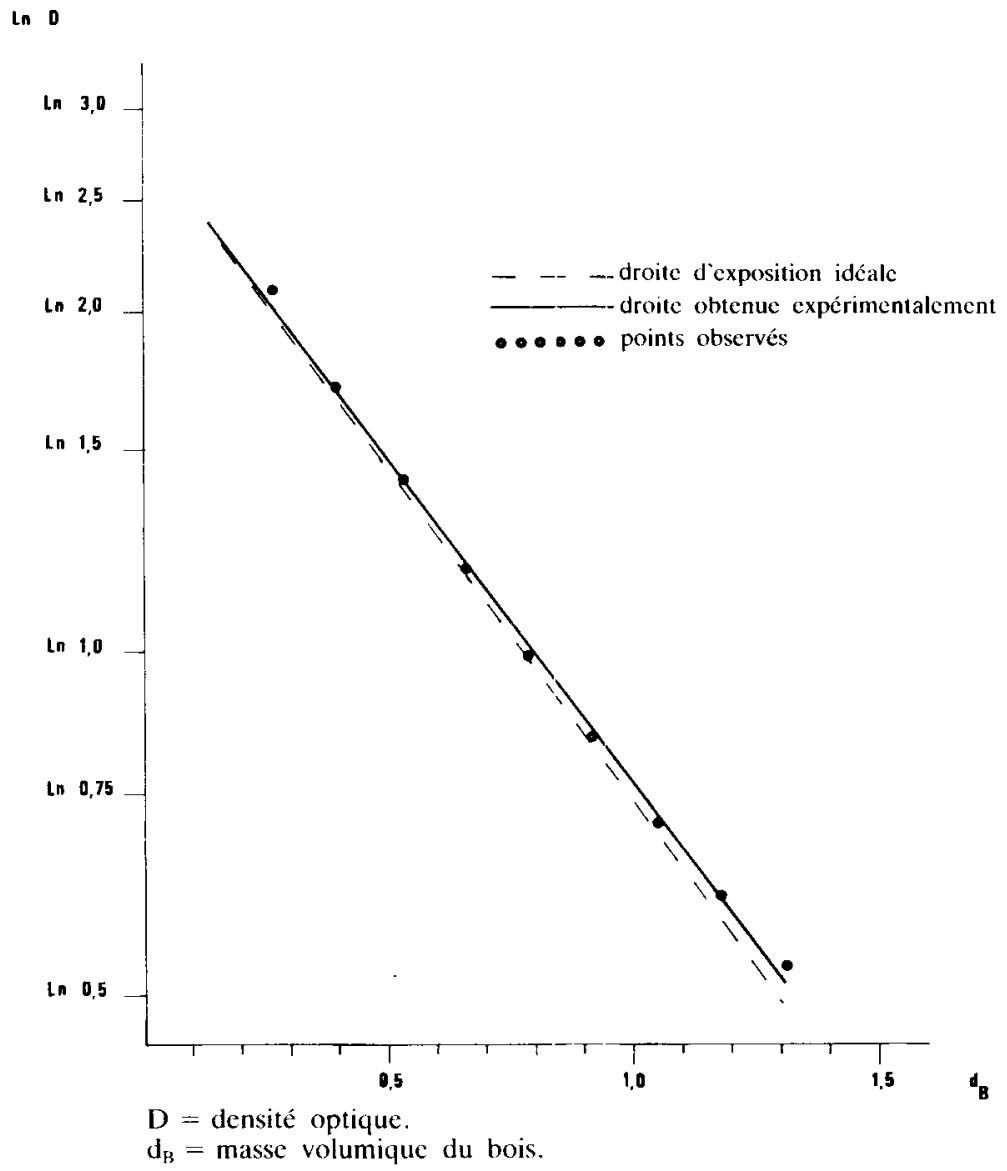

Fig. 2.

Droite d'exposition dans les conditions du test $(24 \mathrm{~mA}, 15 \mathrm{kV}, 25 \mathrm{~mm})$ et droite d'exposition idéale (ligne interrompue).

Exposition's line for the conditions of the test $(24 \mathrm{~mA}, 15 \mathrm{kV}, 25 \mathrm{~mm})$ and the ideal exposition's line (dotted line). The $\bullet$ represent the observed points.

\section{TABlfall 2}

Paramètres d'exposition retenus en fonction de l'épaisseur des échantillons de bois. Exposition's parameters depending on the thickness: thickness $(\mathrm{mm})$, voltage $(\mathrm{kV})$, time (min), intensity $(m A)$.

\begin{tabular}{c|c|c|c}
\hline \hline Epaisseur en mm & Tension kV & Temps (min) & Intensité $(\mathrm{mA})$ \\
\hline 1,5 & 12 & 400 & 24 \\
3,0 & 15 & 24 & 24 \\
6,0 & 22 & 1,5 & 24 \\
\hline
\end{tabular}




\section{Conclusions}

La méthode décrite précédemment a permis de fixer objectivement les paramètres d'exposition optimaux. Quelles que soient les conditions de radiographie, le matériel utilisé et les épaisseurs des échantillons, il nous paraît souhaitable de rechercher cette droite d'exposition théorique de manière à standardiser cette étape de l'analyse microdensitométrique.

L'étalon d'acétate placé sur chaque radiographie permet de contrôler la droite d'exposition lors de l'opération d'étalonnage.

Nous effectuons ce contrôle automatiquement sous la forme d'un test sur le coefficient angulaire et l'ordonnée à l'origine dans le programme microdensitométrique qui a été mis au point au laboratoire.

De plus, les nombreux essais nécessaires pour approcher l'exposition optimale nous ont permis de tester abondamment le modèle théorique décrit au $\S 6.1$. et nous ont incités à l'utiliser désormais, comme base de transformation densité optique-masse volumique bois, en lieu et place du modèle « linéarisé » par l'utilisation d'un coin non linéaire.

Accepté le 23 décembre 1985.

Reçu le 18 juillet 1986.

\section{Summary \\ Determination of optimum $X$-rays exposure parameters in microdensitometry}

This study defines, then formalizes, in microdensimetric analyses of wood, the notion of optimum exposure to X-rays, taking account of technical restraints: characteristics of wood specimen, type of film, standard, source of radiation, exposition parameters (distance « sourcefilm », tension, intensity, time of exposure).

One of the most important problems is the conversion of the film's optical density in wood specific gravity of the material analysed. The described method allows to avoid the use of the continued coin's radiography of the standard, commonly used instead of the optical glass coin of the microdensitometer.

It formalizes a theoric model of exposure conditions. Besides, it shows with an example that it's easy, by experiments, to get this optimum in every particular case, by changing the peak voltage and the exposure time.

Key words: Wood, exposure, density, X-ray, microdensitometry.

\section{Zusammenfassung}

Bestimmung der optimalen Bestrahlungsbedingungen bei der radiographischdensitometrischen Bestimmung der Holzdichte

Durch diesen Versuch wird der Begriff der optimalen Bestrahlung definiert und formuliert, unter Berücksichtigung der Beschränkungen der mikrodensitometrischen Analyse des Holzes. Ausserdem zeigt der Versuch durch ein Beispiel, dass es leicht ist, dieses Optimum empirisch zu 
suchen, in jedem besonderen Fall, wenn man die Bestrahlungsdauer, die Röntgenrohrspannung und den Wärmungsstrom der Kathode entsprechend einstellt.

Schlüsselwörter: Holz, Bestrahlungsbedingungen, Dichte, Röntgenstrahlen, Mikrodensitometrie.

\section{Références bibliographiques}

AgFa-Gevaert, 1969. Radiofilms industriels. Anvers.

Dupont P., 1978. Etude de la densité du bois par analyse radiographique et application à l'épicéa de l'est de la Belgique. Travail de fin d'études, 81 p., Gembloux, Faculté des Sciences Agronomiques.

EVERTSEN J.A., 1982. Interlaboratory standardisation survey. Bull. de liaison de la microdensitométrie du bois, 2 (2), 3-24.

Polge Hubert, 1966. Etablissement des courbes de variation de la densité du bois par exploration densitométrique de radiographies d'échantillons prélevés à la tarière sur des arbres vivants. Applications dans les domaines technologique et physiologique. Thèses, Nancy, Faculté des Sciences de l'Université de Nancy. 